\title{
Electrocardiographic changes in acute ulcerative colitis
}

\author{
C. G. SEMPLE \\ M.B., M.R.C.P.
}

\author{
J. M. Williamson \\ M.B., F.R.C.P. (Glasgow and Edinburgh)
}

University Department of Medicine, Glasgow Royal Infirmary, Glasgow G4 OSF, and Department of Medicine, Royal Alexandra Infirmary, Paisley, Scotland

\section{Summary}

Two young women with acute ulcerative colitis were found to have electrocardiographic abnormalities which returned to normal with clinical improvement and corticosteroid therapy. Clinical and biochemical monitoring failed to reveal any cause for this previously unrecognized association and it is postulated that an aetiological agent or toxin produced by the inflammatory process may have damaged the myocardium or its autonomic supply.

\section{Introduction}

Electrocardiographic (ECG) abnormalities have been described in several diseases not related to the cardiovascular system, for example cholelithiasis (Fitz-Hugh and Wolferth, 1939), head injury (Hersch, 1961) and subarachnoid haemorrhage (Shuster, 1960) but we know of no reported association with ulcerative colitis. Two cases are described of acute ulcerative colitis accompanied by ECG abnormalities which returned to normal as the patient's clinical condition improved with corticosteroid therapy.

\section{Case reports}

Case 1

A 30-year-old female presented with a 4-week history of bloody diarrhoea, nausea and vomiting. A diagnosis of ulcerative colitis had been made from rectal biopsy 4 years previously during a similar illness although she had been symptom-free since then on no drug therapy. Apart from generalized abdominal tenderness and a sinus tachycardia 110 per min, examination was unremarkable.

Haemoglobin was $14.6 \mathrm{~g} / \mathrm{dl}$, WCC $15.2 \times 10^{9} / 1$ and erythrocyte sedimentation rate (ESR) $43 \mathrm{~mm} / \mathrm{hr}$. Estimations of aspartate aminotransferase (SGOT), alanine aminotransferase (SGPT), lactate dehydrogenase (LDH), and electrolytes throughout the course of her illness were normal. A barium enema

Correspondence: Dr C. G. Semple, University Department of Medicine, Glasgow Royal Infirmary, Glasgow G4 0SF.

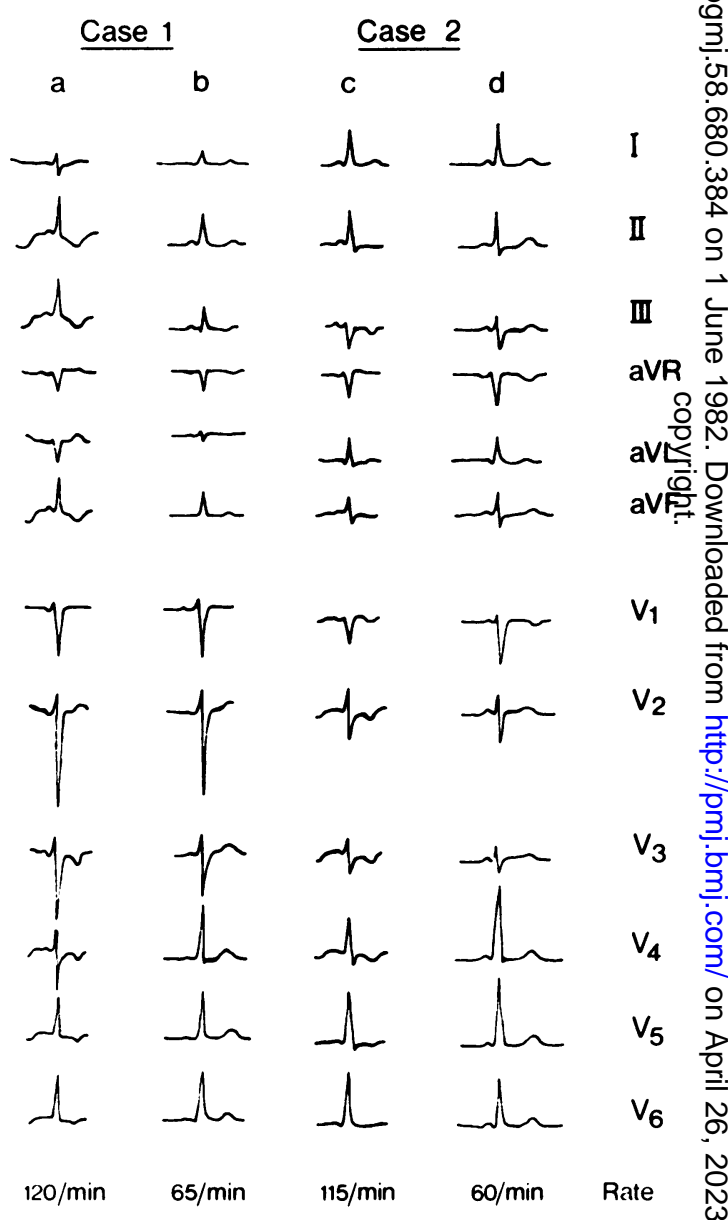

FIG. 1. Electrocardiograms before and after corticosteroid therapy.

showed distal colitis. The ECG on three consecutive 7 days before oral corticosteroid therapy showed a $\stackrel{\circ}{\circ}$ sinus tachycardia with pronounced T-wave inver- 
sion in the infero-lateral leads (Fig. 1 column a, rate 120 per min). Within 3 days of starting oral corticosteroids her ECG had improved and by 14 days it was normal (Fig. 1 column b, rate 65 per min). In addition to improvement in the ST-T segments, the axis of this patient shifted to the left after therapy. She made a full and rapid recovery.

\section{Case 2}

A 32-year-old female gave a one year history of passing four loose stools a day accompanied by the passage of mucus. During the week before admission she had experienced colicky abdominal pain with increasing diarrhoea. Five years previously she had been treated for thyrotoxicosis but was clinically and biochemically euthyroid at the time of presentation. She was taking the contraceptive pill but no other medication. Examination revealed a pyrexial patient with a sinus tachycardia and right sided abdominal tenderness.

Haemoglobin was $13 \mathrm{~g} / \mathrm{dl}$, WCC $16.0 \times 10^{9} / 1$ and ESR $120 \mathrm{~mm} / \mathrm{hr}$. Inflamed rectal mucosa was seen at sigmoidoscopy with biopsy revealing $a$ chronic inflammatory cell infiltrate. A barium enema showed pancolitis with superficial ulceration and pseudopolyp formation. Estimations of electrolytes, SGOT, SGPT and LDH throughout the course of her illness were normal. ECGs on two consecutive days before oral corticosteroid therapy showed a sinus tachycardia with $\mathrm{T}$-wave inversion best seen in the septal leads (Fig. 1 column c, rate $115 / \mathrm{min}$ ). Within 2 days of starting oral corticosteroids her ECG had improved and 9 days later it was normal (Fig. 1 column d, rate 60/min). She made a full and rapid recovery.

\section{Discussion}

Despite their typical clinical presentations, both patients had pronounced ECG abnormalities which reversed with corticosteroid therapy and clinical improvement.
Myocardial ischaemia could account for these abnormalities although the age and sex of our patients must make this explanation unlikely. Moreover there was no clinical or laboratory evidence to suggest this diagnosis or other coexistent pathology such as pulmonary thromboembolism although we recognize that more detailed and invasive investigations would have been required to exclude these diagnoses. Electrolytic disturbances may cause ST-T changes but we have no evidence for biochemical abnormalities in our patients although serum levels poorly reflect intracellular electrolyte concentrations.

ECG abnormalities in subarachnoid haemorrhage are associated with myocardia necrosis (Neil-Dwyer et al., 1978) and may result from increased sympathetic outflow (Neil-Dwyer et al., 1974). A similar mechanism might account for the ST-T abnormalities and persistent sinus tachycardia in our patients. Alternatively some aetiological agent or toxin produced by the inflammatory process might have a deleterious effect on the myocardium or its autonomic supply.

* We conclude that reversible ECG abnormalities may occur in apparently uncomplicated acute ulcerative colitis and that the ECG of such patients should be interpreted with caution.

\section{References}

Fitz-Hugh, T. \& Wolferth, C.C. (1939) Cardiac improvement following gallbladder surgery. Annals of Surgery, 101, 478.

Hersch, C. (1961) Electrocardiographic changes in head injuries. Circulation, 23, 853.

Neil-Dwyer, G., Cruikshank, J., Stott, A. \& Brice, J. (1974) The urinary catecholamine and plasma cortisol levels in patients with subarachnoid haemorrhage. Journal of the Neurological Sciences, 22, 375.

Neil-Dwyer, G., Walter, P., Cruikshank, J.M., Doshi, B. \& O'Gorman, P. (1978) Effect of propranolol and phentolamine on myocardial necrosis after subarachnoid haemorrhage. British Medical Journal, 2, 990.

ShUSTER, S. (1960) The electrocardiogram in subarachnoid haemorrhage. British Heart Journal, 22, 316. 